\title{
Facilitating complex knowledge in design education through design tools
}

\author{
M. Casais \\ CIAUD, Lisbon School of Architecture, Universidade de Lisboa, Portugal
}

\begin{abstract}
Design is a practice-based discipline, which is reflected in its education methodology. In this context, consuming complex knowledge might prove challenging, because designers think and work in a predominantly visual way. However, being able to master precise subjects offers a unique advantage in design, in contrast with a dispersed and superficial approach to knowledge. In this paper, we argue that design education can benefit from using design tools as a didactic way of conveying complex knowledge and making it actionable. To illustrate our proposition, we analysed 14 theory-focused design tools, showing how these convey knowledge and make it usable. In addition, we compared design tools with their respective sources of knowledge to showcase the contrast, proposing a three-part model of knowledge accessibility. Lastly, we describe anecdotal experiences: teaching design classes with and without design tools. The ideas discussed in this paper represent opportunities for further research.
\end{abstract}

\section{INTRODUCTION}

Design is a practice-based discipline, which is reflected in its education methodology, grounded in project development-notwithstanding the textual components present in design history, theory and critique. As such, making use of dense knowledge might prove challenging, because designers think and work in a predominantly visual way, and use visual representation to organize and communicate their thinking (Cross, 1982; Schön, 1983; Lawson, 2005; Wastiels, Schifferstein, Wouters, \& Heylighen, 2013).

The fact that designers are visual thinkers does not mean that they do not benefit from understanding and incorporating knowledge specificity into their practice. On the one hand, multidisciplinarity has become a necessary tenet of our economy, and navigating diverse fields with some fluency and applying that information offers an (apparent) upper hand. On the other hand, it is true that being able to master a precise subject gives a unique advantage in contrast with a dispersed and superficial type of approach (Newport, 2016).

Therefore, we argue, design students can benefit from engaging with specific complex knowledge. This is particularly relevant concerning knowledge that can directly benefit the outcome of design interventions. For example, it is beneficial to deepen knowledge about materials, technology, politics, social issues, users, communities, or society at large, or about predicting or speculating on new and future situations. Its source can be anthropology, engineering, psychology, consumer research, sociology, healthcare, or other fields.

In this paper we propose that design education can benefit from using design tools originating from design research - which articulate design with different fields of knowledge - as an engaging and effective way of conveying complex knowledge and of making it actionable.

Design tools are compact vehicles of data, often with game elements, that deliver methods of working, inspire with ideas or solutions, and summarise complex information in a format that is possible to handle. Such tools have the potential to increase eloquence in intricate matters, by streamlining concepts and theories. They can offer an introduction that demystifies 
the apparent obscurity of complex knowledge, bridging theory and practice through an action-driven medium.

To illustrate our proposition, we conducted an analysis of information-focused design tools (as opposed to tools that offer only practical methodological support) available in physical format. We demonstrate how these convey information and make it accessible and actionable.

In addition, we provide some comparisons of the analysed tools with their respective sources of knowledge, frequently in the form of scientific articles or books, to showcase the stark contrast. Stemming from this comparison, we present a three-part model, based on the levels of accessibility of knowledge to design students.

Lastly, we share anecdotal experiences: teaching classes on design for emotion and wellbeing - knowledge based on psychology — with and without design tools. The ideas discussed in this paper represent opportunities for further research.

\section{DESIGN TOOLS}

Creative thinking tools are aids for creative and problem-solving tasks, using visual and textual stimuli to communicate methods, techniques or strategies. These tools often summarise complex information in the form of booklets, card decks, or digital formats, frequently using game elements.

Two widespread examples of creative thinking tools are the Thinkpak card deck (Michalko, 2006), based on the SCAMPER technique (an acronym for substitute, combine, adjust, modify/ magnify, put to other use, reverselrearrange); and 75 Tools for Creative Thinking (Cordoba Rubino, Hazenberg, \& Huisman, 2013), a box with five card decks (1. Get Started; 2. Check Around; 3. Break It Down; 4. Break Free; 5. Evaluate \& Select) for creative inspiration, also available as a mobile application.

Design tools - a specific form of creative thinking tools - are those developed within design research or practice, articulating diverse fields, such as healthcare, psychology, or consumer research, with design. In general, design tools aim to trigger designers in their process by providing inspiration and information in a flexible way that also allows freedom for them to apply their own understanding in their practice (Sleeswijk Visser, 2009). Design toolkits are a collection of different tools with the same theme/aim.

Several attempts have been made to categorise design tools to understand what these aim to afford in terms of assistance to designers in their processes. Roy and Warren (2018) provided an overview of existing classifications of (card-based) design tools, finding them ultimately focused on small samples and on subjects that arguably fall outside the scope of the design discipline. To tackle this, they proposed their own classification using a sample of 72 cardbased design tools, grouping them under the following themes:

Systematic Design Methods and Procedures;

Creative Thinking and Problem Solving;

Human-Centred Design;

Domain-Specific Methods;

Team Building and Collaborative Working;

and Futures Thinking.

Potentially, any of these tools could be what we designate as information/theory-focused tools, that is, vehicles for summarized complex knowledge from diverse fields that can enrich the design practice in many ways.

To have a more focused understanding of how design tools convey such theory-based, dense information and make it accessible and actionable, we conducted an analysis of different specimens, directed at the knowledge-focused potential discussed above. 


\subsection{Analysis of design tools}

24 design tools were selected through an online search using the terms design tool[s], design method[s], and design toolkit[s]. The search results were not overwhelming, and it was easy to discern examples that fitted the criteria of search, namely that the tool or toolkit would have at least partly a physical tangible component, and that it originated from design research or practice.

An analysis on those 24 tools consisted in checking them against a list of elements which could potentially be used to argue for our proposition. The aim of this preliminary analysis was firstly to grasp a range of formats, navigation styles, and presentation of information, and secondly to uncover information/theory-focused tools. Those constituent elements were:

1. Origin: Which design/education institution developed and launched the tool;

2. Format: Which elements constitute the toolkit (e.g. canvas, posters, cards);

3. Icons: Does it use symbols, icons, pictograms to structure navigation;

4. Images: Does it use images to provide examples of context or depict other related things;

5. Infographics: Does it use graphic representations of information in combination with text;

6. Linear text: Does it contain linear text;

7. Non-linear text: Does it contain non-linear text, e.g. lists, networked text/words, wordclouds;

8. Action-focused: Does it explain a method of working and/or prompt the designer to work in a directive way;

9. Information/theory-focused: Does it convey theoretical information or knowledge about a topic;

10. Colour coding: Does it use colour to structure navigation;

11. Graphs/diagrams: Does it use graphs and/or diagrams to illustrate or explain content;

12. Game elements: Does it contain explicit game instructions or elements, or does it tacitly invite the user to get involved in a gamified experience (due to its components, set of instructions, need for partnering, etc.);

13. Digital components: Does it contain digital components such as applications, photo albums, etc.;

14. Other relevant elements: Does it have other relevant elements, such as instruction manual;

15. Objectives: Description of the objectives as provided in the tool itself.

Following the preliminary analysis, we shifted our attention to the focused analysis of information/theory-focused tools, that is, those tools whose primary aim was to convey theoretical knowledge from other fields to enrich the design practice at any level (e.g. understand specific users, or people in a global sense, understand situated contexts, predict outcomes of designs, speculate about future scenarios).

\subsection{Results}

The analysis of the 24 design tools (see annex) showed that their origin was not indicative of their content, i.e., a tool developed by an education institution did not aim to, necessarily, convey complex knowledge. However, we did find examples of this type of theory-based tool from design research (see TU Delft examples).

While we aimed for tools with at least one tangible component, the majority had either one or more. Most analysed tools contained a card deck, canvas, posters, or booklets. One third (8) had additional digital components, such as web-based guides. However, all analysed tools were able to be downloaded or accessed digitally, and subsequently printed or ordered in printed format.

Colour frequently played a role in organizing and hierarchizing information. In addition, the tools largely contained both text and image, often both linear and non-linear text.

The tools aimed to provide information and/or methodological instructions, as well as design cases that illustrated these methods or techniques. Specifically, we found that the objectives could be divided into four types: (1) summarizing theoretical knowledge; (2) 
providing inspiration/displaying design cases; (3) understanding the user; and (4) providing methodological support.

Lastly, we observed over one third (9) with specific game elements or game-like instructions. We found 14 design tools in our sample that corresponded to the criterion of being information/theory-focused (see annex).

A further analysis of these 14 tools revealed that these have certain traits in common, namely:

Action: accompanying the theory, the tools presented a methodological component which allowed the knowledge to be put into practice.

Format: the tools were presented in a size that is easy to handle (in the design studio or in class), and in a portable format, such as a booklet or card set.

Pictorials: images supplement linear and non-linear text and provide a more vivid illustration of a given phenomenon.

Language: the theory is often accompanied by eliciting conditions (this happens when...) presented in simple terms.

Text: linear text is kept short and turned into graphs or illustrated when appropriate, text is highlighted or enhanced to hierarchize information.

\subsection{A model of information accessibility: Three levels of communication and understanding}

When we compare the information/theory-focused design tools with their respective sources, the stark contrast between how the information is presented becomes apparent. Taking the case of two design tools-Positive Emotion Granularity Cards (Yoon, Pohlmeyer, \& Desmet, 2015) and SIM toolkit (Casais, Mugge, \& Desmet, 2016), we can explore how information about a certain topic is accessible in different ways to design students.

We begin by looking at the original source that led to the design research work. In the two illustrated cases bellow (Figure 1 and 2) the original sources are from the field of psychology and are presented in the form of books and scientific articles. We designate this as level 1 of
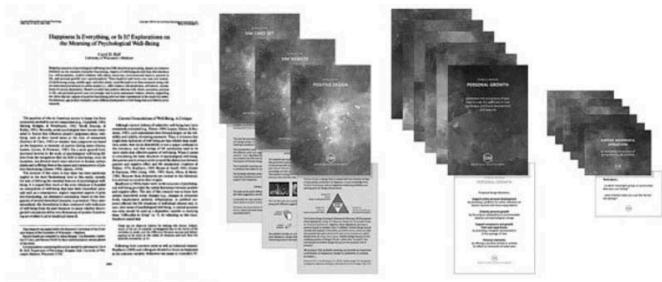

Figure 1. On the left side, a scientific article about the determinants of psychological well-being (Ryff, 1989); on the right side the SIM toolkit (Casais et al., 2016), based on Ryff's theory.

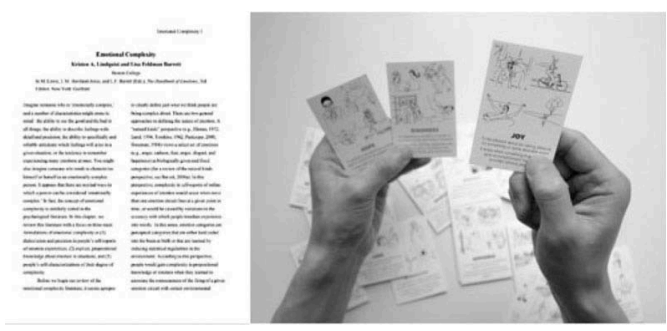

Figure 2. On the left side, a book chapter about emotional complexity (Lindquist \& Barrett, 2008); on the right side, the Positive Emotional Granularity Cards (Yoon, et al. 2015), based on Lindquist and Barret's theory. 


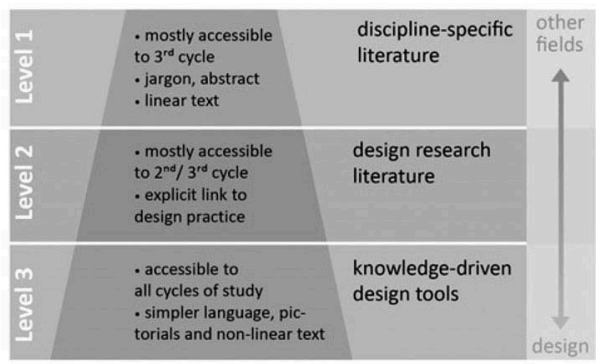

Figure 3. Model of information accessibility.

accessibility, a type of knowledge and presentation of information that is most commonly accessible to $3^{\text {rd }}$ cycle design students (and eventually also $2^{\text {nd }}$ cycle students). This is due to the use of discipline-specific terminology, abstract reasoning, and dense sections of linear text which are not particularly appealing to visual thinkers.

On an intermediate level, we find the design research from which the design tools were developed; we call this level 2 of accessibility, more approachable by $2^{\text {nd }}$ and $3^{\text {rd }}$ cycle design students. In the given cases, the sources are also in the form of scientific articles and books. At this level we find a link from the original source-discipline to the field of design, showing explicitly how there is relevance for design practice, which renders this type of reading more appealing and digestible by $2^{\text {nd }}$ cycle students. However, we often find some dislike for the abundance of linear text, density of information, and abstract reasoning.

At level 3 of accessibility we find the design tools, widely accessible for all cycles of education. At this level, as uncovered in the previous section, knowledge is made available through easy language, pictorials accompanying linear and non-linear text, actionable directions, and portable or manageable formats.

In Figure 3 we find a model of information accessibility summarizing and illustrating the three levels of communication and understanding.

\section{SOME ANECDOTAL EXPERIENCES}

In numerous occasions, the author had the opportunity to use design tools in design education. However, these were not specifically set up to evaluate the effectiveness of this modality in conveying complex knowledge. Rather, design tools were used to complement a traditional type of design class, in which there was a lecture about theoretical knowledge, a moment to apply the theory in project-based exercises, and a discussion moment. The design tools were used to exemplify the application of certain theories into methodological aids, or in discussions and in short exercises.

Nevertheless, these anecdotes reveal the potential impact of information/theory-focused design tools in design education and are encouraging for the pursuit of further investigation on the subject. Two interesting cases of teaching design students about human emotions and wellbeing are described below, one without design tools and one with.

\subsection{Case 1: Teaching emotions with traditional didactic instruments}

The first case occurred in a workshop setting, part of a semester-long course, in the $1^{\text {st }}$ semester of the $2^{\text {nd }}$ year of the $2^{\text {nd }}$ cycle of study, with 5 groups of students (each group with 4 to 5 students). Students attended an engineering design master programme from an industrial design engineering faculty.

Immediately prior to the workshop, students attended a lecture on the topic of human emotions and subsequently were asked to read one level 1 publication (a book chapter) and two 
level 2 publications (a research article and a book chapter) (see Figure 3). Then, they were asked to prepare for the workshop by analysing a product from the user's perspective applying the learned theoretical concepts, and to summarize and visualise their findings.

During the workshop, students had one hour to redesign the product in accordance to their findings, one hour to discuss and present, and 5 minutes to receive focused feedback from the teacher.

During the contact hours of the workshop, the degree of difficulty to communicate about the concept of human emotions and their applicability was high. A suitable analogy to describe the situation is trying to make oneself understood by a foreign person that does not speak the same language or share the same basic gestures or symbols. Furthermore, students found it incredibly difficult to incorporate something as abstract as emotions in their designs.

\subsection{Case 2: Teaching wellbeing with theory-focused design tools}

The second case occurred in a workshop setting, part of a two-week elective course, in the $1^{\text {st }}$ semester of the $1^{\text {st }}$ year of the $2^{\text {nd }}$ cycle of study, with 3 groups of students (each group with 4 to 5 students and 1 student facilitator per group). Students attended an engineering design and a strategic design master programme, from an industrial design engineering faculty.

The workshop preparation occurred in class and was focused on setting up a creative session within an innovation team, aiming to expand their knowledge of creative techniques and later experience process consulting with real cases - so no focus was given to the theme of wellbeing or related content.

The workshop was setup and facilitated by the students themselves, and the author provided the real case, acting as a client. The case description provided to the students was to think of alternative uses for a theory-focused card set about wellbeing, a topic stemming from the field of psychology. Possible uses could have been, for example, in communication with end-users, clients or design teams, in mental healthcare or counselling, etc. Students had 1:30 hour of creative session, followed by one hour of presentation and discussion.

While this was not a class on human wellbeing, the students' task implied that they understood the theoretical themes being discussed through the theory-focused design tool in order to iterate on the tool's applications in diverse contexts.

To realise whether and how well students understood the topic, the author observed the exploration process of the groups, watched the presentations, and discussed with them. Together, these elements demonstrated the students' remarkable comprehension of the theoretical content in a short time-span. Students also mentioned the simplicity and straightforwardness of the design tool in explaining the topic.

\section{DESIGN TOOLS AS APPROPRIATE DIDACTIC INSTRUMENTS}

Examples of commonly recognised didactic resources can be books, articles, lectures, videos, and workshops. Tools, in the sense that we describe here, are not commonly used as an inclass educational instruments, for the discovery of new topics or deepening of content.

However, we argue that for different reasons this modality presents a great potential particularly in design education. In this section we list some compelling motives in trying to directly answer the question: why should we consider design tools appropriate didactic instruments for design education?

\subsection{Designers think visually}

Information designer Francis Miller, a practitioner focused on simplifying learning processes (Miller, 2016-2019), proposes a methodology for the treatment of complex information, which he calls multi-level content. Based on Christopher Alexander's ideas, multi-level content is 


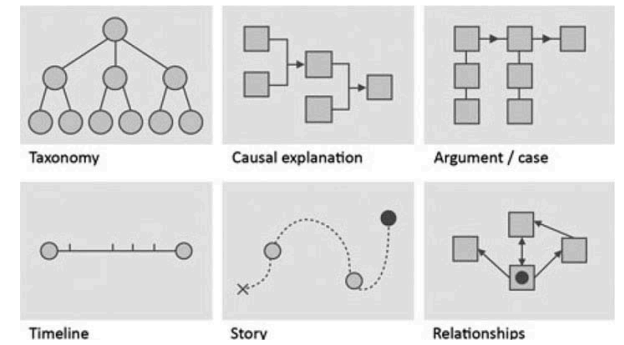

Figure 4. Several types of knowledge structures (based on Miller, 2018, p. 13).

defined as the provision of content over multiple levels of detail ranging from the big picture summary down to a granular detail (Miller, 2018).

Miller argued for his concept of multi-level content by indicating that "making sense of any knowledge requires understanding the structure of the explanation. However, neither spoken or written words on their own are very effective at communicating structure" (Miller, 2018, p. 2).

Clarifying his statement "all knowledge has structure" (p. 13), Miller identified several types of knowledge (Figure 4), some of which are explicit in text, while others are implicit. In large information vessels, such as books, there can be many different types of structures; this means that the challenge is threefold: understanding a (potentially implicit) knowledge structure through a limiting medium like linear text in sequential blocks, uncovering the links between several knowledge structures, and grasping what is being told in an overview. Multilevel content is thus proposed as a solution.

The way Miller presents the knowledge structures in pictorial form is, in itself, a form of multi-level content. Explaining each knowledge structure concept is done succinctly, in a straightforward way, leaving room for other layers of information to be added. The image itself serves to illustrate how striking visualization can work for certain reader profiles, particularly in our case, design students.

Designers, as visual thinkers, can potentially find such simplifications valuable, because they allow the easy and prompt application of ideas into practice. Providing summarized information (linear and non-linear text) with pictorial stimuli provides a more compelling mental image of concepts, concept links, and respective theories, catering especially well to a visual thinking audience.

\subsection{Creating mental images supports learning}

Design tools typically provide information through both pictorial means and verbal means, often in combination. This was corroborated by our tool analysis, wherein all design tools had visual cues (images, colours) or a combination of visual and textual cues. Such multidimensional representation of information is easier to grasp and apply compared to text alone (Santos, Pereira Neto, \& Neves, 2019).

The dual-coding theory (Paivio, 1986/1990) hypothesized that people code information through two distinct coding channels - a visual channel, also called analogue coding; and a verbal channel, also called symbolic coding. These two separate systems processes input differently, images are processed by the visual coding channel, whilst words have a particular path according to their nature: "concrete words are processed by both systems, whereas abstract words are processed primarily by the verbal system" (Kounios \& Holcomb, 1994, p. 804).

This corroborates our proposal that design tools are situated at a third level of accessibility (Figure 3), a wider and more approachable level of language, format and presentation, that can serve the purpose of conveying complex knowledge in simplified and concrete terms aided by pictorials, which in turn support the creation of mental images for theoretical concepts. 
The creation of mental images improves information retention, storage, and retrieval, therefore supporting learning, since a given concept can be recalled by either code, verbal or visual, providing a better chance of remembering (Reed, 2007).

\subsection{Synthesis makes knowledge (more) actionable}

Some design tools were developed because, while certain "academically oriented design guidelines and frameworks" have potential, they are long, dense, use discipline-specific language, and thus become difficult to apply in practice (Hornecker, 2010; Deng, Antle \& Neustaedter, 2014).

Design tools may provide, through the way they synthesize information, a potential solution for this. They summarize dense information and deconstruct it in different elements, such as definitions, application examples and anecdotes, eliciting conditions, behavioural manifestations, illustrating theories with the aid of graphs, diagrams and pictorials, etc. This synthesis was observed on all analysed tools, and while some were more information dense than others, overall, they summarised somewhat complex knowledge - for example, from psychologyinto straightforward and succinct content.

This synthetic display of information potentially stimulates connections that lead to the structured or serendipitous finding of certain routes for design, or to novel solutions to design problems, or even to the uncovering of surprising opportunities and links within the context of work or study.

\subsection{The physical format makes people interact differently}

In her studies on design practitioners, Rogers (2004) found that "a frequently cited complaint was that designers wanted more guidance and ways of communicating about theories and techniques" (p. 39), suggesting that language and format were also an issue.

We encountered several design tools available in digital formats (webpages, video, etc.), making them updatable, easily accessible, and offering a dynamic type of interaction. However, the printed format offers certain advantages that might be diluted with intangibility.

Specifically, printed information makes people interact differently, and "demonstrate[s] a propensity for manipulation by people, modifying communication effect" (Neves, 2019). Nevertheless, the printed format alone does not guarantee engagement.

The comparison of the original source of knowledge with its summarized, visual, and actionable modality - the tool - showcases potential it can have in motivating design students, as discussed above (see Figure 1 and 2).

The advantages of physical elements of design tools, such as card sets, have to do with navigation, hierarchization, flexibility - for evaluation, pairing or comparison, with the ability to provide an overview, and freedom to apply or interpret their use in different ways (e.g. Deng et al., 2014; Casais et al., 2016; Yoon et al., 2016).

Another important aspect associated with tangibility is the possibility of having game elements. This can be used as a strategy to entice design students to engage and learn or apply the tool's contents.

\subsection{Figure captions}

Always use the Figure caption style tag (10 points size on 11-points line space). Place the caption underneath the figure (see Section 5). Type as follows: 'Figure 1. Caption.' Leave about two lines of space between the figure caption and the text of the paper.

\section{CONCLUSIONS}

While conclusions about the effectiveness of theory-focused design tools in a design education setting might be premature at this point, some preliminary thoughts might be considered. 
From the analysis of design tools, we learned that design tools make dense knowledge streamlined, actionable and accessible; and that they present a lot of information in a small and portable size that can be used in multiple ways (particularly card sets). Furthermore, this modality tends to communicate with images and concrete text which helps create strong mental images and aids with better learning. Tools illustrate dense knowledge with various modes of communication: symbols, icons, graphs and diagrams, eliciting conditions, behavioural manifestations, anecdotes, pictures, strategies, solutions, etc.

From the comparison of the design tools and their sources of knowledge, we developed a three-part model of information accessibility. This model summarizes three levels of communication and understanding that design students use, namely: level 1 - the knowledge from other fields other than design, often resorting to discipline-specific complex language, linear text and abstract reasoning; level 2 - the knowledge from design research that articulates design with other fields, makes evident the relevance of such knowledge to design practice but often remains obscure and abstract, communicated through linear text; level 3 - the streamlined, simplified, and actionable version of the knowledge, the design tool, more widely accessible to students.

Further research on the topic could focus on interviewing design teachers that already use such aids in education setting, or those that face the challenge of teaching design students' knowledge from other fields, and together with them set up studies to verify whether the potential effectiveness and engagement that is proposed in the current paper holds true.

\section{REFERENCES}

Casais, M., Mugge, R., \& Desmet, P.M.A. (2016). Design with symbolic meaning for user happiness card set $(S I M)$. Delft: Delft University of Technology. ISBN/EAN: 9789461866387.

Cordoba Rubino, S., Hazenberg, W., \& Huisman, M. (2013). 75 Tools for Creative Thinking. Booreiland. ISBN: 978-90-6369-275-9.

Cross, N. (1982). Designerly ways of knowing. Design Studies, 3(4), 221-227.

Deng, Y., Antle, A.N., \& Neustaedter. C. (2014). Tango Cards: A card-based design tool for informing the design of tangible learning games. In: Proceedings of the 2014 conference on Designing interactive systems DIS'14: Crafting Design, (695-704), Vancouver, June 21-15, 2014. New York, NY: ACM Press.

Hornecker, E. (2010). Creative idea exploration within the structure of a guiding framework: The Card Brainstorming Game. In: Proceedings of the fourth international conference on Tangible, embedded, and embodied interaction TEI'10, (101-108), Cambridge, MA, January 24-27, 2010. New York, NY: ACM

Kounios, J. \& Holcomb, P.J. (1994). Concreteness effects in semantic processing: ERP evidence supporting Dual-Coding Theory. Journal of Experimental Psychology: Learning, Memory, and Cognition. 20 (4), 804-823.

Lawson, B. (2005). How designers think: The design process demystified (4th ed.). Oxford, UK: Architectural Press.

Lindquist, K. A., \& Barrett, L. F. (2008). Emotional complexity. In L. F. Barrett, M. Lewis, \& J. M. Haviland-Jones (Eds.), Handbook of emotions (3rd ed., 513-530). New York, NY: Guilford Press.

Michalko, M. (2006). Thinkpak: A brainstorming card deck. Ten Speed Press. ISBN: 978-1-58008-772-8.

Miller, F. (2016-2019). Making knowledge easier to understand, remember and implement. Retrieved from http://www.francismiller.com/

Miller, F. (2018). Organising knowledge with multi-level content: Making knowledge easier to understand, remember and communicate. Retrieved from http://www.francismiller.com/organising_knowl edge_paper.pdf

Neves M. (2019). Design for (Inter)Actions: A teaching experience in graphic design. In: F. Rebelo \& M.M. Soares (Eds). Advances in Ergonomics in Design AHFE 2018: Advances in Intelligent Systems and Computing, (Vol. 777, 422-431). Springer.

Newport, C. (2016). Deep Work. Rules for focused success in a distracted world. New York, NY: Grand Central Publishing.

Paivio, A. (1990). Mental representations. A dual coding approach. New York, NY: Oxford University Press. (Original work published 1986). 
Reed, S.K. (2007). Cognition. Theory and applications (7th ed.). Belmont, CA: Thomson Wadsworth.

Rogers, Y. (2004), New theoretical approaches for human-computer interaction. Annual Review of Information Science and Technology, 38(1), 87-143.

Roy, R., \& Warren, J. (2018). Card-based tools for creative and systematic design. In: C. Storni, K. Leahy, M. McMahon, P. Lloyd, \& E. Bohemia (Eds.). Proceedings of the Design Research Society conference DRS2018: Design as a Catalyst for Change, (Vol. 3, Sec.10, Tools of Design, 1075-1087). Limerick, June 25-28, 2018. London: Design Research Society.

Ryff, C.D. (1989). Happiness is everything, or is it? Explorations on the meaning of psychological well-being. Journal of Personality and Social Psychology, 57(6), 1069-1081.

Santos C., Neto M.J.P., Neves M. (2019) The Influence of Infographics in Accessing Information: Multidimensionality in Visual Representation and Configuration of Different Media. In: F. Rebelo \& M.M. Soares (Eds). Advances in Ergonomics in Design AHFE 2018: Advances in Intelligent Systems and Computing, (Vol 777, 497-508). Springer.

Schön, D. (1983). The reflective practitioner: How professionals think in action. New York, NY: Basic Books.

Sleeswijk Visser, F. (2009). Bringing the everyday life of people into design. (Unpublished doctoral dissertation). Delft University of Technology. Delft, The Netherlands.

Three-cycle system. (2016, September) Retrieved from http://www.ehea.info/page-three-cycle-system

Wastiels, L., Schifferstein, H. N. J., Wouters, I., \& Heylighen, A. (2013). Touching materials visually: About the dominance of vision in building material assessment. International Journal of Design, $7(2), 31-41$.

Yoon, J., Pohlmeyer, A.E., \& Desmet, P.M.A. (2015). Positive Emotional Granularity Cards. Delft, Delft University of Technology. ISBN: 978-94-6186-440-6. 